\title{
The quest for new physics with the Physics Beyond Colliders programme
}

\author{
Joerg Jaeckel', Mike Lamont ${ }^{2}$ and Claude Vallée $\mathbb{1}^{3 凶}$
}

In recent years, interest in complementary methods to high-energy-frontier colliders to investigate the physics of elementary particles and forces has grown. This development is driven by the so-far negative results of searches for new particles with high masses at the Large Hadron Collider, and by theoretical attempts to account for neutrino masses and to solve cosmological puzzles such as dark matter, dark energy and the matter-antimatter asymmetry in the Universe. Traditionally, CERN has hosted a wealth of accelerator and non-accelerator projects below the high-energy frontier, in which more than a thousand physicists are currently involved. The Physics Beyond Colliders (PBC) study was launched three years ago to explore the future of this field. Here we give an overview of the various PBC proposals, ranging from explorations of the dark sector to precision measurements of strongly interacting processes. The methodology employed to compare the reach of those projects - based on a set of common benchmark models - has raised interest in the collider, neutrino and non-accelerator communities and may provide a comprehensive overview of how the parameter range for physics beyond the Standard Model should be explored in the worldwide context.

F undamental particle physics finds itself currently in the interesting position of being absolutely certain that there must be physics beyond the current Standard Model (SM), describing elementary particles and the weak, electromagnetic and strong forces, while at the same time facing the challenge that it seems exceedingly hard to find ${ }^{1}$. Convincing evidence from cosmology suggests that $95 \%$ of all the matter and energy in the Universe consists of dark matter (DM) and dark energy, which are not described within the SM, although the vast majority of experiments on Earth agree to astonishing precision with SM predictions. Two ways exist to reconcile beyond Standard Model (BSM) physics with a nonobservation in present experiments: new particles could be either very massive or very weakly interacting with the $\mathrm{SM}^{2-4}$.

\section{Theoretical motivation}

Before its discovery, the Higgs boson mass was expected in the range around $100-1,000 \mathrm{GeV}$ from consistency considerations of the SM at energies above $1 \mathrm{TeV}$ (ref. ${ }^{5}$ ). The situation has now fundamentally changed because the SM, with the Higgs boson of around 125 $\mathrm{GeV}$, gives no clear indication of where new physics might be found. The quest for BSM physics requires a vast range of particle masses and couplings to be explored (Fig. 1a). High-energy colliders such as the Large Hadron Collider (LHC) and proposed machines such as the International Linear Collider, the Compact Linear Collider or the Future Circular Collider are crucial for the direct observation of putative very massive particles. Complementary experiments with low energy but high intensity or precision, as discussed within the PBC programme, search for particles with either low mass and weak couplings, or very high mass, the latter becoming accessible through their indirect effects on observables (see also refs. ${ }^{3,4,6}$ for more information on worldwide efforts in this area). Although there is currently no decisive evidence of deviations from the SM in highintensity or precision experiments, a few tantalizing hints require further investigation, as discussed in Box 1.

The cosmological puzzles, as well as the current anomalies (Box 1), could be explained by the existence of a dark sector - new 'hidden particles' that carry no SM gauge charges and are therefore either weakly interacting with ordinary matter or/and very long lived. Within the PBC study, a set of benchmark models representing popular and well-motivated extensions of the SM were defined. These models are based on the idea of portals to a dark sector (see, for example, refs. ${ }^{6,7}$ for a discussion and further references) that are the simplest interactions between SM particles and the new hidden particles. In particular, the PBC study focuses on the following models.

Dark photons with and without additional DM particles. The interactions of the SM arise from three gauge groups giving rise to the photon for the electromagnetic interaction, the $W^{ \pm}$and $Z^{0}$ bosons for the weak interaction and the gluons for the strong interaction. The photon is the simplest force carrier because it does not carry charge and therefore does not couple to itself. A potential, minimal extension of the SM is therefore via an additional 'dark photon' that does not couple to massive SM particles. In consequence, the only and potentially very weak interaction of the dark photon with a SM particle is via small mixing with the photon, resulting in a small conversion probability of the dark photon into a photon. This simplest extension of the SM gauge interactions by an additional photon-like particle has many applications in DM model building.

Heavy neutral leptons. All charged fermions of the SM exist in a left- and a right-handed version (naively, handedness refers to the direction of the spin with regard to the direction of movement). The uncharged neutrinos have only been observed as left-handed particles. However, it seems only natural for neutrinos to have righthanded counterparts. Similar to dark photons, these right-handed neutrinos would interact only via small mixing with the SM neutrinos. Right-handed neutrinos can play a role in explaining the small neutrino masses, DM, and also the dominance of matter over antimatter in the Universe.

New scalar particles. Generally speaking, scalar particles, carrying neither spin nor charge, are the simplest imaginable particles. The most direct interaction of additional scalars with the SM would be via mixing with the only other known fundamental scalar, that is,

University of Heidelberg, Heidelberg, Germany. ${ }^{2}$ CERN, Meyrin, Switzerland. ${ }^{3}$ Centre de Physique des Particules de Marseille, Marseille, France.

凶e-mail: vallee@cppm.in2p3.fr 
a

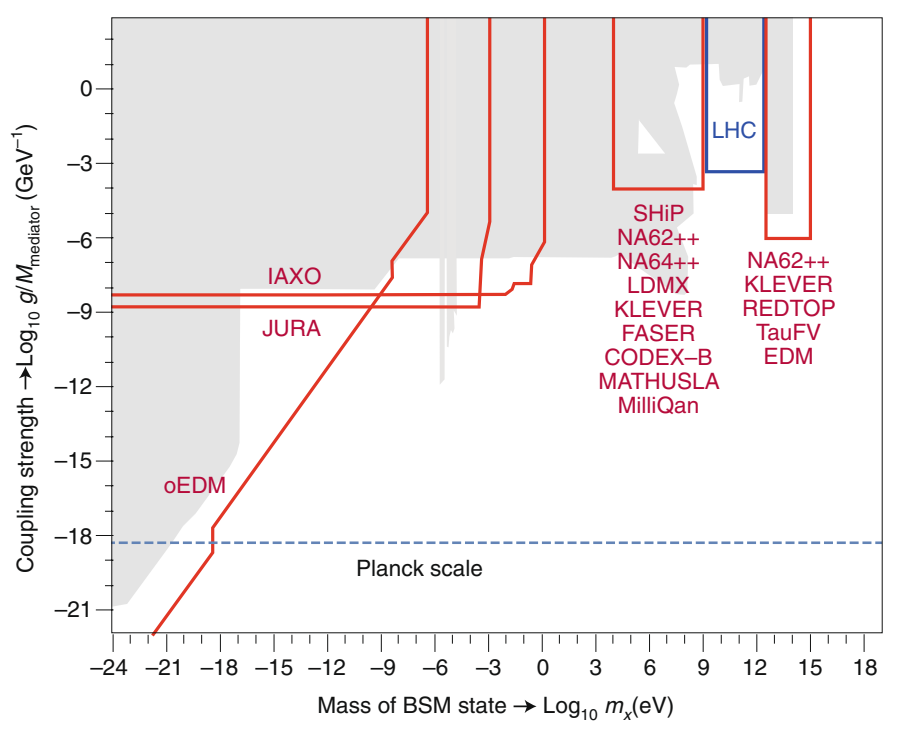

b

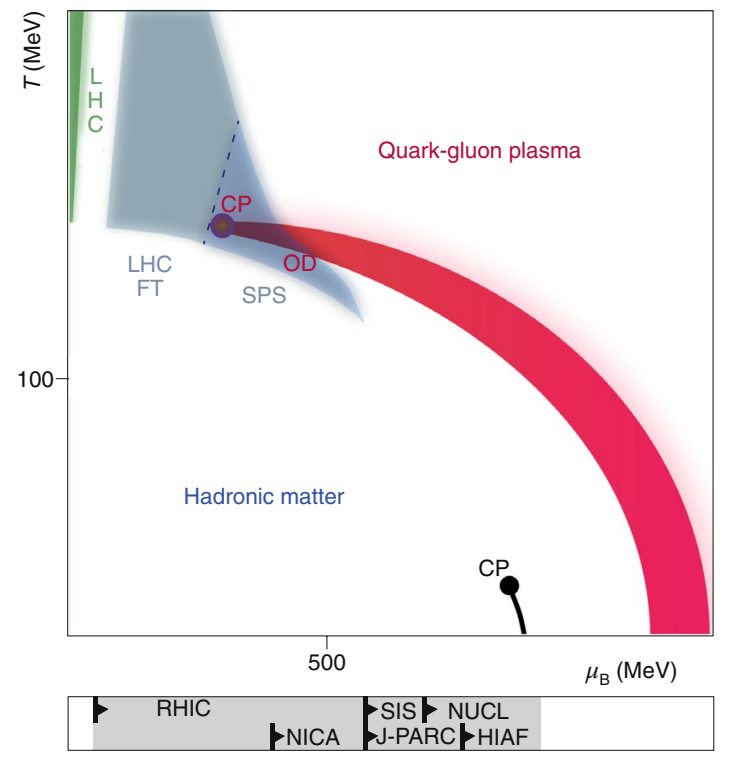

Fig. 1 The potential parameter space covered by PBC projects in BSM searches and QCD studies. a, Schematic overview of the BSM areas targeted by different PBC projects as function of the mass of the lightest BSM particle $m_{x}$ and its coupling strength to the SM $g / M_{\text {mediator }}$ The Planck scale corresponds to the coupling strength of the gravitational force. The shaded area corresponds to domains that have already been probed (for details and caveats see refs. ${ }^{1,6}$ ). Precision experiments (right), beam dump experiments (middle) and low-energy experiments (left) are compared to the reach of the LHC (blue region). The proposed experiments for long-lived particle searches are included for comparison to beam dump experiments. See main text and Table 1 for details of the experiments. b, Conjectured QCD phase structure as function of the baryonic potential $\mu_{\mathrm{B}}$, roughly quantifying the excess of quarks over antiquarks, and the temperature $T$. The numbers on these axes indicate the typical scales of interest for $\mu_{\mathrm{B}}$ and $T$. At high temperature, quarks and gluons are deconfined from the usual hadronic matter made of quark bound states into a quark-gluon plasma. The region of the onset of deconfinement is labelled OD. The expected critical point (CP) delimitates a region of first order phase transition on the right (red band) from a second order phase transition on the left. At low temperature, increasing the density induces a phase transition from a gas of hadrons into a liquid of hadrons (short black line, ending in another critical point). The situation at high temperature is less clear than at low temperature and subject to theoretical and experimental investigations. The achievable values of $\mu_{\mathrm{B}}$ and $T$ are determined by the atomic numbers of the beams and targets and by the beam energies. The domains covered by the Super Proton Synchrotron (SPS), the final pre-accelerator of the LHC delivering beams to fixed target experiments, and the LHC fixed-target heavy-ion experiments (LHC FT) are indicated in dark and light grey, respectively. The reach of the current LHC experiments is illustrated in green. The scale at the bottom provides a comparison to the reach of other facilities worldwide, namely the Relativistic Heavy lon Collider (RHIC) in operation in the US, the Nuclotron Based Ion Collider Facility (NICA) and the Heavy lon Synchrotron (SIS) in construction in Russia and Germany, respectively, and the lower energy ion facilities in operation in Russia (NUCL), Japan (J-PARC) and China (HIAF). Figures adapted with permission from PBC BSM and QCD working groups: $\mathbf{a}$, ref. ${ }^{1} ; \mathbf{b}$, ref. ${ }^{47}$.

the Higgs boson. If coupled to the Higgs boson, additional scalars could feature in solutions to the so-called hierarchy problem, which refers to the puzzle of why the energy scale of the weak interactions is so much smaller than that of gravity. New scalar particles have also been discussed in relation to DM.

Axions and axion-like particles. Axions and axion-like particles are (pseudo-)scalar particles. In contrast to the new scalars discussed above, they are special in the sense that they are thought to arise as remnants of an underlying symmetry. For this reason, axions and axion-like particles are expected to be light and their interactions strongly suppressed. Axions have been proposed to explain the parity (mirror) symmetry of the strong force, demonstrated to a high precision by the observed null electric dipole moment (EDM) of the neutron. Axion-like particles are also good portals to DM and are even promising DM candidates themselves.

While these benchmark models are not an exhaustive list of DM and dark sector models, they provide a useful way to compare the sensitivities of different experiments and see how they complement each other as shown in Fig. 1a. They also provide a map of promising target areas suggested by the open problems of particle physics and the current hints of experimental anomalies.
In addition to the need for new physics, it has to be recognized that even the SM is not yet fully understood. In particular the theory of the strong interactions, quantum chromodynamics (QCD), requires a better description of its behaviour at high temperatures and densities, notably in the phase transition between bound and free quarks. Here, experiments discussed within the PBC programme explore unique kinematic regimes available at the CERN complex, complementary to other facilities worldwide as illustrated in Fig. 1b.

Within QCD, the dynamics of real or virtual multi-quark systems, so-called hadrons, is difficult to compute precisely with perturbative methods, because the coupling of the strong force increases with distance. The interpretation of experiments relying on hadron beams, such as the LHC, or of observables that are sensitive to virtual quark exchanges, such as B-hadron decays or the anomalous magnetic moment of the muon defined via the $g$-factor as $a_{\mu}=$ $(g-2)_{\mu} / 2$, is therefore often limited by QCD theoretical uncertainties. Despite the rapid progress of non-perturbative computations (for example, lattice, renormalization group or Schwinger-Dyson) of QCD-related observables (see for example, ref. ${ }^{8}$ for $(g-2)_{\mu}$ ), direct experimental measurements of the hadronic structure and of hadronic virtual processes will remain crucial in the foreseeable future to overcome these limitations. 


\section{Box 1| Anomalies as potential hints for new physics}

A small number of measurements and observations might indicate deviations from SM predictions at the level of two to four standard deviations $(\sigma)$. These hints may be the first signs of an impending discovery of new physics and provide an enticing incentive for theoretical model building. In turn, these theoretical models then allow one to devise new, and more crucially, different tests of the interpretations of these anomalies. However, it is important to note that the significance usually only covers the statistical uncertainties and the known systematic errors. Therefore, independent experiments to measure the quantities in question are desirable to confirm the veracity of the anomalous observations. In the following, we briefly discuss the most intriguing hints.

\section{B-anomalies}

Decays of B-mesons show several deviations of $\sim 2 \sigma-3 \sigma$ from the SM prediction ${ }^{51}$. Notably, in the SM model the gauge couplings of different leptons $(e, \mu$ or $\tau)$ are expected to be equal. Yet the measurements indicate a potential violation of this expected universality. Suitable extensions of the SM (for example, based on effective field theory) allow for an explanation without violating other experimental constraints (see, for example, ref. ${ }^{52}$ ).

$(g-2)_{\mu}$

The size of the magnetic dipole moment of the muon can be calculated and measured with exquisite precision. It is therefore a classic precision test of the SM. The reference measurement ${ }^{53}$ indicates a $3 \sigma-4 \sigma$ deviation from the SM expectation (notably both theoretical and experimental uncertainties are similar in size). Explanations could arise from quantum corrections due to weak scale $\sim 100 \mathrm{GeV}$ particles with $\mathrm{O}(1)$ couplings or from much lighter $\sim 10-100 \mathrm{MeV}$ particles with $\mathrm{O}\left(10^{-3}\right)$ couplings. A minimal example of the latter type - a dark photon ${ }^{54}$ - provides an interesting example of where the theoretical model led to an experimental test. This test conducted in a fixed target experiment and at low energy colliders ruled the dark photon out as a possible explanation. Other simple model ${ }^{55,56}$ in this area remain viable. A new experiment ${ }^{57}$ is currently taking data at Fermilab in the US with the promise to reduce the experimental uncertainty on $(g-2)_{\mu}$ by a factor of four.

\section{Beryllium anomaly}

Experiments at the Atomki facility in Hungary have investigated electron-positron pairs emitted in the decay of excited states of ${ }^{8} \mathrm{Be}$ and ${ }^{4} \mathrm{He}$ (refs. ${ }^{48,49}$ ). In both cases, they find more events than expected at large angles between the electron and the positron. A potential explanation could be a new boson (see, for example, refs. ${ }^{48-50}$ ) that is emitted at low velocity from the nucleus and subsequently decays into an electron-positron pair. In the rest frame of this particle, the electron-positron angle would be $180^{\circ}$ and, due to the low velocity, the angle in the laboratory frame would only be slightly compressed. This indicates a boson mass of $\sim 17 \mathrm{MeV}$ (refs. ${ }^{48-50}$ ).

\section{Cooling anomalies}

Several astrophysical systems such as white dwarfs and horizontalbranch stars, as well as supernova remnants, seem to cool slightly faster than expected. This could indicate an extra energy loss via the emission of extremely weakly coupled, light (masses below $100 \mathrm{keV}$ ) particles. If such particles are produced in the interior of a star, they immediately leave. This is in contrast to, for example, photons that are scattered multiple times on their way to the surface, thereby reducing the energy loss via this route. For this reason, extremely weakly coupled light particles can lead to significant energy loss despite a relatively small interaction strength (see for example, ref. ${ }^{58}$ and references therein for an overview).

\section{Gamma-ray transparency}

High-energy gamma-rays propagating through the Universe are depleted by collisions with the extragalactic background light, creating electron-positron pairs. Observations find that there are more high-energy photons than expected, indicating a way around this depletion mechanism. One option is that in the presence of magnetic fields some photons convert into very light bosons, notably axion-like particles. These then travel without being absorbed by pair creation and finally convert back to photons (see ref. ${ }^{58}$ and references therein for a discussion).

\section{CERN infrastructure specifics and opportunities}

CERN is currently the only particle physics laboratory worldwide devoted to the exploration of the high-energy frontier - the LHC remaining the priority for the coming two decades. Other large laboratories such as Fermilab and Jefferson Lab in the US, and KEK in Japan, host lower-energy, high-intensity projects primarily devoted to precision physics. Despite its current focus on the high-energy frontier, CERN has always demonstrated unique strengths for complementary experiments beyond colliders.

The CERN domains of specific interest for the PBC study include:

- The LHC beams themselves, which provide by far the highest particle energies of up to $6.5 \mathrm{TeV}$ for protons, and for which several novel applications such as fixed-target physics and the generation of high-intensity gamma-ray beams were proposed.

- The Super Proton Synchrotron (SPS) shown in Fig. 2. The SPS acts both as the LHC main proton injector, and as a versatile machine delivering high-intensity proton beams with energies up to $450 \mathrm{GeV}$ in parallel to several experiments. The key facility from a PBC perspective is the North Area, which hosts a number of fixed-target experiments with widely varying physics

goals. The SPS also periodically delivers ions to the LHC and users in the North Area.

- The technology of the current accelerator systems, and the vigorous ongoing research and development for future accelerators, which influenced the discussions of novel experiments such as those for axion searches.

Lower-energy facilities, such as the ISOLDE radioactive ion beam facility, the Neutron Time Of Flight (n-TOF) pulsed neutron beam facility and the antimatter factory, have been recently upgraded to run over the next decade and were therefore not considered within the PBC study. The CERN expertise in the physics of low-energy machines was, however, crucial in scrutinizing some of the PBC projects.

Proposals building on the LHC beams. The current LHC experiments cover only a small region of the QCD phase diagram. As indicated in Fig. 1b, fixed-target physics with LHC protons and ion beams would extend the reach to a new kinematic domain that was up to now covered by the Relativistic Heavy Ion Collider (RHIC) with a limited statistical precision. Instead of extracting the LHC 


\section{Table 1 | List of projects submitted to the PBC study group}

\begin{tabular}{|c|c|c|c|}
\hline Experiment & Physics case & Status & Time scale \\
\hline NA61++ & Charm in QCD phase transition & Operational/upgrade studies & Near \\
\hline NA60++ & Caloric curve of QCD phase transition & Feasibility study & Medium \\
\hline COMPASS ++ & QCD dynamics & Operational/upgrade studies & Near \\
\hline MUonE & Hadronic vacuum polarization for $(g-2)_{\mu}$ & Prototype/tests with beam & Near \\
\hline LHC FT (gas storage cell) & QCD dynamics and phase transition & Installation/further studies & Near \\
\hline KLEVER & Ultra-rare decays of neutral kaons & Feasibility studies & Medium \\
\hline TauFV & Ultra-rare decays of tau leptons & Design study in progress & Long \\
\hline REDTOP & Ultra-rare decays of eta meson & Proposal & Medium \\
\hline NA64++ & Dark photon searches with electron and/or muon beam dump & Operational/upgrade studies & Near \\
\hline LDMX & Dark photon searches & Design study in progress & Medium \\
\hline BabyIAXO/IAXO & Axion search (helioscope) & $\begin{array}{l}\text { Conceptual design/ } \\
\text { prototyping }\end{array}$ & Medium \\
\hline JURA & Axion and axion-like particle searches & Exploratory studies & Long \\
\hline VMB@CERN & Vacuum magnetic birefringence & Letter of intent/studies & Medium \\
\hline Facility & Beam type & Status & Time scale \\
\hline BDF & High intensity $400 \mathrm{GeV}$ protons for SHiP and TauFV & Design study complete & Medium \\
\hline eSPS & $16 \mathrm{GeV}$ electrons & Design study in progress & Medium \\
\hline nuSTORM & Neutrino beam from a muon storage ring for cross-section measurements & Feasibility study complete & Long \\
\hline EDM ring & Polarized proton storage ring for EDM measurement & Feasibility study complete & Medium \\
\hline
\end{tabular}

The level of maturity (status) and approximate time-line (time scale) for each experiment/facility is indicated as in ref. ': near term, before 2025; medium term, 2025-2030; long term, after 2030. See main text for discussion of the individual projects.

beams into new experimental halls, internal targets close to the LHCb and ALICE detectors, which have a large forward acceptance, would be implemented. This approach offers the advantage of minimizing the required investments while relying on existing collaborations and detectors. The method was already pioneered by the $\mathrm{LHCb}$ experiment with the injection of gas close to the $\mathrm{LHCb}$ collision point. Within the scope of the PBC programme, a systematic study ${ }^{9}$ of fixed target experiments was carried out, in particular regarding more sophisticated systems such as gas storage cells ${ }^{10}$, polarized targets ${ }^{11}$, and the deflection of protons in the beam halo with bent crystals onto an internal target ${ }^{12}$. The gas targets can explore the QCD phase transition and hadron structure in a new kinematic regime, whereas the crystal extraction approach aims at the measurement of the magnetic and electric dipole moment of short-lived baryons.

Additionally, the gamma factory ${ }^{13}$, relying on a novel concept, proposes to use the large relativistic boost of partially stripped ions stored in the LHC to convert a laser photon beam into a high-intensity gamma-ray beam. A leap of up to seven orders of magnitude in intensity could be gained compared to current gamma-ray facilities, opening potential new avenues ${ }^{14}$ to domains as diverse as fundamental quantum electrodynamics, nuclear and neutrino physics with rare processes, or dark sector searches. A first milestone was passed within the PBC framework with the successful storage and acceleration of partially stripped ions in the LHC, paving the way to a proof-of-principle demonstration of the gamma factory.
A road ahead at the SPS. In preparation for the high-luminosity phase of the LHC, a major upgrade of the existing LHC injector complex is ongoing ${ }^{15}$, with the principal aim of delivering the requested higher number of particles per bunch and beams with a low transverse emittance. The upgrades are also expected to significantly reduce losses in both the Proton Synchrotron (the accelerator stage before the SPS) and the SPS for the beams that are delivered to the North Area. This will pave the way for new high-current experiments in the North Area. The upgrades of the LHC injector complex together with the termination of the operation of the CERN neutrino beam to Gran Sasso, Italy, in 2012 hold promise for a new general-purpose high-intensity facility at CERN.

The new SPS facilities considered in the first instance are beam dumps. In a beam dump experiment, a beam is fully absorbed within a target in order to search for new weakly-interacting or long-lived particles escaping the target. Those hidden particles can be detected with different approaches, depending on the beam structure as illustrated in Fig. 3. High-intensity pulsed beams are suitable for detection of the new particle's decay products, or of the recoil particle from its elastic re-scattering within a heavy target. In so-called active instrumented beam dumps, lower-intensity continuous beams are required to observe invisible particle production from missing energy or momentum in the products of individual beam particle collisions.

The flagship PBC beam dump project is the beam dump facility (BDF) based on the SPS high-intensity $400 \mathrm{GeV}$ proton beam. 


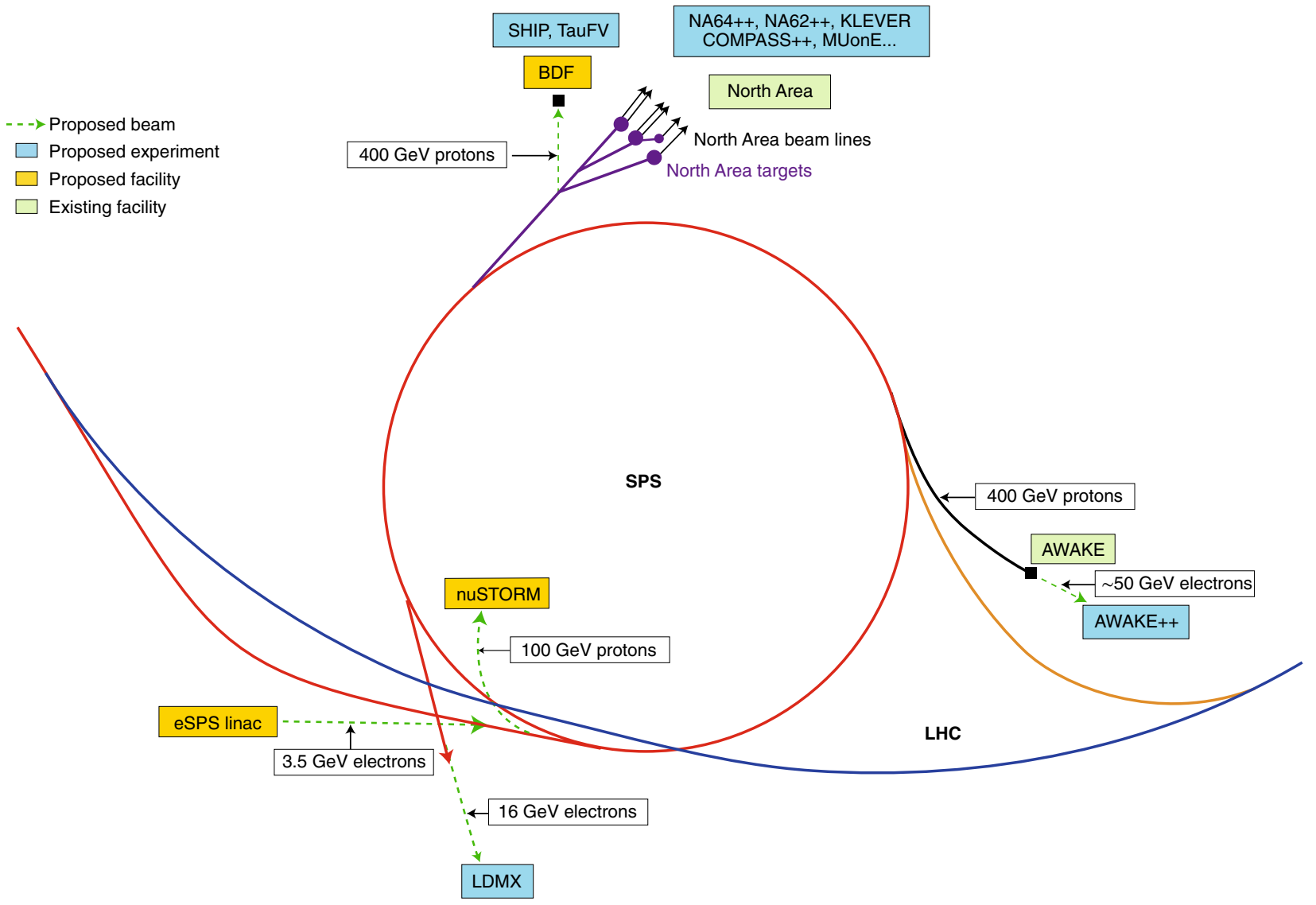

Fig. 2 | Schematic overview of the SPS showing existing and proposed facilities. Solid lines indicate existing beam lines, dashed lines refer to proposed new beams, and proposed new experiments are shown in blue. See main text for descriptions of the projects.

Within the PBC study, the design of the BDF has reached the comprehensive design stage ${ }^{16}$. The overall layout in the SPS North Area is fully defined and critical aspects such as target design and radioprotection are well advanced. In particular, studies were performed to minimize proton losses when the beam is extracted from the SPS. These studies were critical to maximize the total intensity available to the BDF, and will also allow improved delivery of conventional North Area beams. In the first instance, exploitation of the facility is envisaged to be for the Search for Hidden Particles (SHiP) experiment, looking for weakly-interacting and long-lived particles, and the TauFV experiment, which uses a fixed-target geometry to search for flavour violation in tau-lepton decays as a sign for BSM physics.

Experiments rooted in research and development for novel acceleration techniques. In addition to the proton beam dump proposal discussed above, electron beam dump experiments building on CERN research and development for new acceleration techniques are discussed. The eSPS project ${ }^{17}$ foresees injecting $3.5 \mathrm{GeV}$ electrons into the SPS using X-band accelerator structures developed during intensive research and development for the Compact Linear Collider. The electrons would be subsequently accelerated to $16 \mathrm{GeV}$ and then slowly extracted to a new experimental facility devoted to DM searches using the missing momentum method (Fig. 3c). The SPS also delivers beam to the Advanced Proton Plasma Wakefield Acceleration (AWAKE) research project. Following the successful demonstration of the AWAKE concept in its first phase ${ }^{18}$, and assuming success of the next phase planned for the coming years, a new high-intensity medium-energy pulsed electron beam could be implemented in the former CERN neutrino project area to search for new particles (AWAKE++ $)^{19}$.
Another proposed SPS-based facility is the Neutrinos from Stored Muons (nuSTORM) project, which is designed to deliver a neutrino-nucleus scattering programme using beams of neutrinos from the decay of muons confined within a storage ring. The SPS would provide the primary proton beam for muon production and offers a credible location for fast extraction towards a new experimental area ${ }^{20}$.

Developments in acceleration techniques are also expected to foster further experiments that do not rely on a particle beam. CERN has traditionally hosted a number of non-accelerator experiments exploiting accelerator technologies for specific new physics searches, such as the CAST axion helioscope ${ }^{21}$ using a spare LHC dipole magnet. A systematic survey of this domain has identified several technologies, among them high-field magnets, radio-frequency cavities, cryogenics, and vacuum and optical techniques, as particularly suited for exchange of expertise between CERN and outside laboratories to develop new experimental non-accelerator projects $^{22}$.

\section{PBC projects potential in the worldwide context}

The PBC experiments build on the unique features of the facilities discussed above to explore the whole spectrum of possible new particle masses and couplings (Fig. 1a). They also foresee new measurements of the QCD phase transition (Fig. 1b) and of the internal dynamics of hadrons. A full list of PBC project proposals is given in Table 1 , indicating their maturity and approximate time scale. The projects will be discussed in the following.

QCD measurements. Some of the PBC QCD-oriented projects aim at providing new fundamental insights on the strong phase 
a

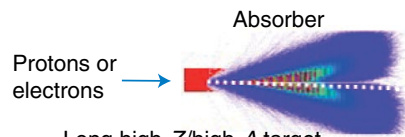

Long high- $Z$ /high- $A$ target

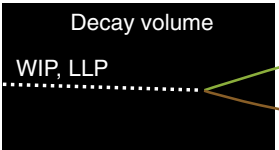

Spectrometer

(O)

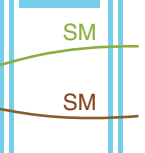

$\odot$

b

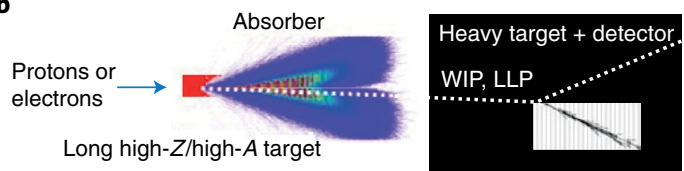

c

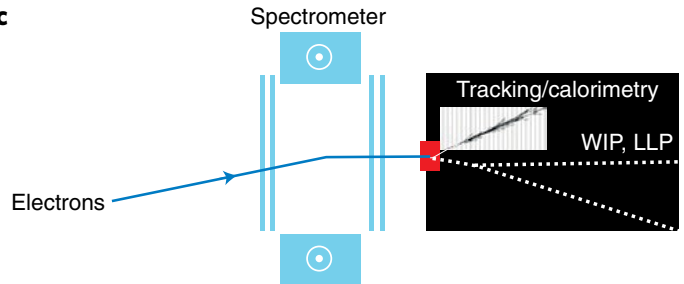

Fig. 3 | Detection methods of hidden particles in beam dump experiments. A beam of electrons or protons impinges on a long target made from a material with high proton $(Z)$ or mass number $(A)$, which then absorbs the beam fully. a, The weakly-interacting particles (WIP) or long-lived particles (LLP) escaping the beam dump are expected to decay within a vacuum vessel into visible SM particles measured by a spectrometer. $\mathbf{b}$, The weaklyinteracting or long-lived particles escaping the beam dump recoil from elastic scattering in a heavy target and are detected from the recoil electron or nucleus of their elastic interaction. c, Weakly-interacting or long-lived particles, which carry a certain energy and momentum, are identified from an apparent violation of energy or momentum conservation. Within an instrumented beam dump, the momentum or energy of the incident electron is compared to that of all decay products, a visible deficit meaning that the missing momentum or energy was carried away by particles invisible to the detector. The method requires a precise control of the incident beam particles and full hermeticity of the instrumented setup. Options in $\mathbf{a}$ and $\mathbf{b}$ can be best realized with a high-intensity pulsed beam; option in can be best realized with a continuous beam. Credit: Richard Jacobsson, CERN

transition using fixed targetion collisionsat theSPSandLHC(Fig. 1b). Highlights at the SPS include an upgrade of the NA61 experiment $(\mathrm{NA} 61++)$ for high statistics measurements of heavy quark production, that is, charm production, in the phase transition region ${ }^{23}$, and a revival of the past NA60 experiment $(\mathrm{NA60++)}$ for the measurement of the phase transition caloric curve with low-energy muon pairs ${ }^{24}$

Measurements of hadronic systems of interest for both the understanding of the strong force and new physics searches are also foreseen. The current flagship QCD programme of the SPS, COMPASS, has defined a new long-term program as a QCD facility (COMPASS ++$)^{25}$. The MUonE experiment ${ }^{26}$ proposes a novel method, based on muon-electron elastic scattering, in order to reduce the dominant theoretical uncertainty on the $(g-2)_{\mu}$ prediction. The project benefits from the availability of a unique high-energy and high-intensity muon beam at CERN, but is experimentally very challenging. However, this would provide a timely input to the new $(g-2)_{\mu}$ experiments in progress in the US and planned in Japan. There is also a proposal to revive the past DIRAC experiment (DIRAC++) for improved measurement of low-energy QCD dynamics with hadronic atoms at the SPS.

Among the wealth of new measurements opened by LHC fixed-target collisions ${ }^{27}$, one important goal is the improvement of the knowledge of the high-momentum quark content of the proton - of particular interest to maximize the reach of searches for new high-mass particles in the high-luminosity phase of the LHC. Altogether, the physics reach of fixed-target setups at the LHC will crucially depend on the maximal fixed-target collision rates, which can be accumulated without affecting the main high-luminosity LHC collision physics goals.

Rare decays and precision measurements. The SPS is currently at the forefront of precision physics connected to the second quark generation, with the measurement of ultra-rare decays of charged kaons into a pion and two neutrinos - in progress by the NA62 experiment ${ }^{28}$. KLEVER $^{29}$ proposes to extend such measurements to neutral kaons, promising complementary results to NA62 which would provide a full picture as the similar measurements in the bottom-quark sector. The project is, however, very challenging since the only visible decay product is a neutral pion, which is more difficult to discriminate from background than a charged pion. The phasing of KLEVER with NA62 will depend on the NA62 results because the observation of a deviation for charged kaons would clearly require investigating neutral kaons, but also on the progress of a competing experiment, $\mathrm{KOTO}^{30}$, in Japan, and on the future evolution of the B-anomalies (Box 1) and their possible explanations.

Similar measurements in the leptonic sector could be made at the new BDF by intercepting a small fraction of the incident beam upstream of the dump within the TauFV detector, which would be devoted to searches for ultra-rare tau-lepton decays. In particular, the SM-forbidden decay of a tau lepton into three muons could be measured with a precision higher than that expected from the

Fig. 4 | Expected sensitivities of PBC projects to a selection of dark sector channels. Limits are shown as function of the hidden particle mass $m_{A^{\prime}}$ or $m_{N^{\prime}}$ and its coupling $\varepsilon$ or $U_{\tau}$ to the SM particle. Regions within $(\mathbf{a}, \mathbf{c})$ or above $(\mathbf{b})$ the curves are expected to be excluded at $90 \%$ confidence level in case no signal is detected. The shaded areas correspond to already excluded regions. See main text for description of the PBC experiments. $\mathbf{a}$, PBC projects searching for dark photons visible decay modes (Fig. 3a,b). The vertical red bar labelled ${ }^{8}$ Be corresponds to the region which would explain the current Be anomalies (see Box 1 and refs. ${ }^{48-50}$ ). The two FASER curves correspond to the expected accumulated luminosities for the accepted phase one and a possible extension on the whole high-luminosity phase of the LHC, respectively. The luminosity indicated for the NA64 green curve corresponds to the NA64++ upgrade. The purple curve labelled NA62 corresponds to the proposed beam dump operation of the experiment (NA62++). $\mathbf{b}$, PBC projects searching for dark photons based on missing energy or momentum (Fig. 3c). BaBar and Belle II are experiments at low energy electron-positron colliders completed in the US and starting in Japan, respectively. PADME, VEPP3 and MMAPS are active target experiments in operation or preparation on low-energy positron beams at the Frascati Laboratory in Italy, Novosibirsk in Russia, and Cornell in the US. The luminosity indicated for the NA64 green curve corresponds to the NA64++ upgrade. c, PBC projects for searches for heavy neutral leptons coupled exclusively to the tau lepton. The two FASER curves correspond to the expected accumulated luminosities for the accepted phase one and a possible extension on the whole high-luminosity phase of the LHC, respectively. The purple curve labelled NA62 corresponds to the proposed beam dump operation of the experiment (NA62++). CHARM was a fixed target experiment at CERN and DELPHI was one of the four experiments of the high-energy electron-positron collider LEP at CERN. The region labelled 'BBN' is excluded by Big Bang nucleosynthesis, and the region labelled 'See Saw' is excluded in case the heavy neutral leptons are expected to account for the small neutrino masses via the so-called see-saw mechanism. Figure adapted with permission from: ref. ${ }^{6}$, under CC BY 3.0. 
BELLE II experiment ${ }^{31}$ in Japan. Such a project would be welcome to augment the physics reach of the BDF. There is also a plan to measure ultra-rare decays of low mass hadrons (REDTOP), but the experiment requires a continuous high-intensity low-energy proton beam which would be more easily implemented for example, at Fermilab in the US.
In the low-energy domain, the possibility of measuring the proton EDM via the spin precession of polarized protons in an all-electrostatic storage ring - a novel method initially proposed in the US - has been studied in depth by the CPEDM collaboration ${ }^{32}$. The leading hadron EDM measurements worldwide are currently made with neutrons, and they target a precision close to $10^{-28} \mathrm{e} \mathrm{cm}$ in the

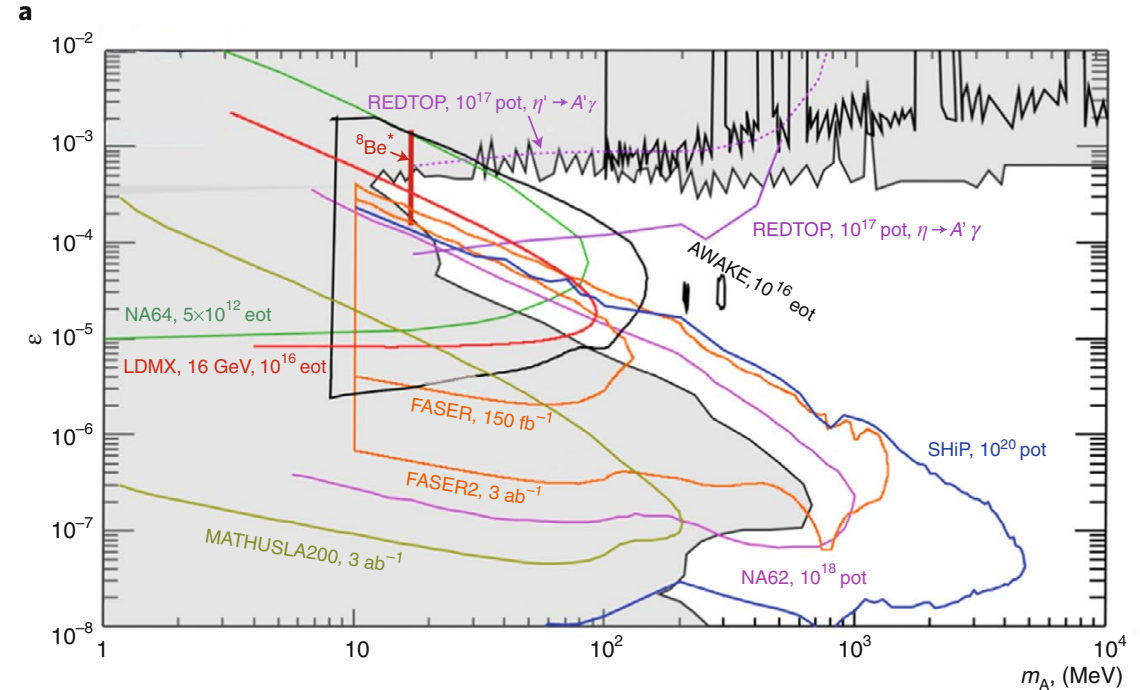

b

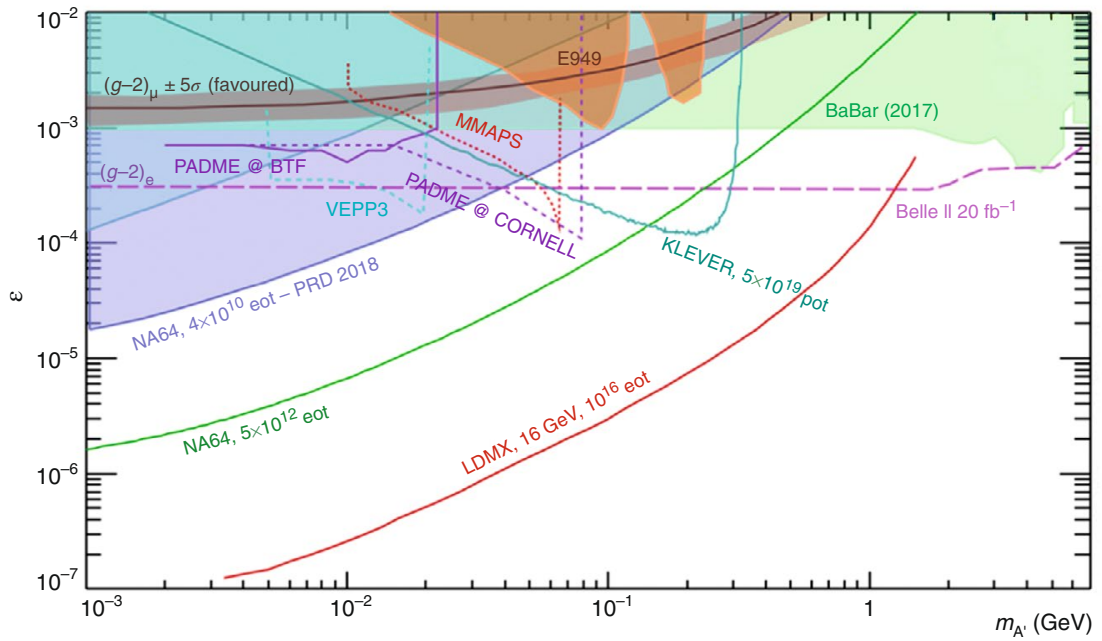

C

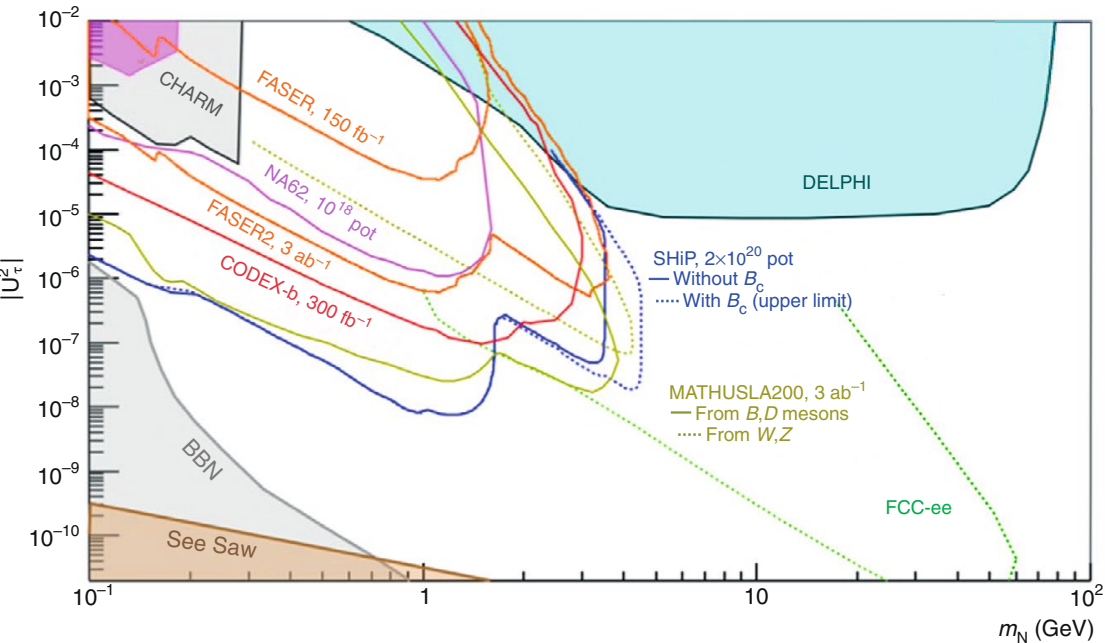


coming decade. The novel proton EDM ring offers the prospects of increasing the precision by an order of magnitude compared to the neutron EDM. However, this represents a challenging leap of ten orders of magnitude compared to the precision of previous storage ring EDM experiments. One critical aspect is the control of parasitic magnetic fields within the ring. Careful scrutiny by the project team has identified the need for a prototype ring, possibly at the site of the COSY deuteron storage ring in Jülich, Germany, to validate the technique. The polarized protons stored in the EDM ring may also be directly sensitive to an ambient DM field made of axionlike particles. Those could produce an oscillating EDM (denoted as oEDM in Fig. 1a) detectable through a time series analysis of the data or by scanning the beam's spin-rotation frequency in search of a resonance with an axion-like particle mass in the range from $10^{-6} \mathrm{eV}$ down to $10^{-24} \mathrm{eV}$ (refs. ${ }^{33,34}$ ).

Dark sector. Following a first generation of beam dump experiments in the 1970s and 1980s, performed mainly as by-products of experiments addressing other topics, there is currently a revived interest in the method to search for a dark sector. Several dedicated experiments are ongoing worldwide, notably with electron beams in the US and at CERN, where the NA64 experiment ${ }^{35}$ relies on the missing energy method introduced in Fig. 3c. Similarly, the proposed Light Dark Matter Experiment (LDMX) ${ }^{17}$ is also based on the missing momentum method (Fig. $3 \mathrm{c}$ ), and would receive electron beam from the eSPS facility. LDMX has also a shorter-term implementation opportunity at the Stanford Linear Accelerator Center (SLAC) in the United States, pending approval of an electron beam extraction line at the Linear Coherent Light Source (LCLS-II), which is an upgrade of SLAC's free-electron laser facility presently under construction. The proposed AWAKE++ pulsed electron beam could serve a beam dump experiment with a detector sensitive to visible dark photon decays ${ }^{19}$. The proposed SHiP detector $^{36}$, a dual spectrometer sensitive to both the visible decay (Fig. 3a) and re-scattering (Fig. 3b) of hidden particles such as long-lived or weakly-interacting ones, relies on the proton BDF and is shielded from the dump by a state-of-the-art muon filter.

The sensitivities of these projects to the benchmark models described above have been compared using comprehensive simulations of the various setups. For completeness, the comparison included the dedicated projects for long-lived particle searches proposed at the LHC: FASER ${ }^{37}$, a recently approved small detector located $480 \mathrm{~m}$ from the ATLAS collision point, CODEX-b ${ }^{38}$, milliQan $^{39}$ and MATHUSLA ${ }^{40}$. The results are shown for dark photons and one example of heavy neutral lepton in Fig. 4. However, dark scalars and axion-like particles convey similar messages.

For invisible dark photons (Fig. 4b), the NA64 intensity upgrades $(\mathrm{NA} 64++)$ will explore significant new parameter space in the short term, whereas LDMX (if realized at the proposed eSPS) has the potential to cover most of the parameter space, which could explain the DM abundance in the Universe when assuming that dark photons decay into a stable neutral DM particle. An AWAKE-based beam has an interesting potential for searches of dark photons in the visible mode as shown in Fig. 4a, but most of the accessible parameter space is likely to be covered by the foreseen competition in the coming decade $^{6}$. SHiP has the highest long-term reach in the low-coupling/ high-mass domain of the explored region as indicated in Fig. 4a.

For heavy neutral leptons (Fig. 4c), there is a significant amount of new parameter space to be explored in the short term by a proposed part-time beam-dump configuration of NA62 (NA62++) and by FASER. In the longer-term, SHiP has the highest reach, even when compared with dedicated projects to search for long-lived particles at the LHC, establishing heavy neutral leptons as a flagship channel of the experiment.

It is worth noting that the CERN SPS offers an optimal configuration for a proton beam dump in terms of energy and intensity.
In particular, the $400 \mathrm{GeV}$ proton energy provides copious hadron yields for the full mass spectrum including B-hadrons. An exploratory study performed for axion-like-particle searches ${ }^{41}$ has shown that fixed-target beam dumps with higher-energy proton beams from the LHC or Future Circular Collider beams would not open significant new domains to hidden sector searches. The masses and couplings targeted by SPS fixed target experiments are also fully complementary to those explored by present and future highenergy-frontier colliders: this is visible in Fig. $4 \mathrm{c}$ for heavy neutral leptons, where the electron-positron stage of the Future Circular Collider sensitivity covers a higher mass region than SPS beam dumps, and was confirmed for all types of dark portals by a comprehensive study performed for the European Strategy Group ${ }^{42}$.

Current non-accelerator experiments at CERN searching for a dark sector include the CAST helioscope ${ }^{21}$ and the OSQAR experiment ${ }^{43}$, both of which use LHC magnets to look for axions produced in the Sun or in the laboratory, respectively. Next generation projects are under consideration. The International Axion Observatory (IAXO) helioscope $\mathrm{e}^{44}$ builds on CAST and the latest CERN magnet technologies, and its precursor BabyIAXO is in the approval stage at the Deutsches Elektronen Synchrotron (DESY) centre in Germany. The JURA project also emerges as a potential new long-term project dedicated to laboratory axion searches, combining the forefront optical technologies of the DESY ALPS II experiment ${ }^{45}$ and the high-field magnets developed at CERN for the high-luminosity LHC and Future Circular Collider. Figure 1a indicates the potential gains in sensitivity versus current limits for JURA and IAXO. Based on similar technologies than ALPS II, the VMB project at $\mathrm{CERN}^{46}$ has been initiated within PBC with the aim to test quantum electrodynamics in the presence of high magnetic fields with a first measurement of vacuum magnetic birefringence. Such projects are good examples of how collaboration of CERN with external nonaccelerator laboratories can be fostered for the mutual benefit of all contributors.

The PBC programme offers many unique physics opportunities, but resource and beam limitations restrict the number of proposals that can be pursued. The consideration of an integrated plan for future developments in the field in Europe rests now with the European Strategy for Particle Physics Update.

Received: 9 September 2019; Accepted: 13 February 2020; Published online: 6 April 2020

\section{References}

1. Alemany, R. et al. Summary report of physics beyond colliders at CERN Preprint at https://arxiv.org/abs/1902.00260 (2019).

2. Jaeckel, J. \& Ringwald, A. The low-energy frontier of particle physics. Ann. Rev. Nucl. Part. Sci. 60, 405-437 (2010).

3. Hewett, J. L. et al. Fundamental physics at the intensity frontier. Preprint at https://arxiv.org/abs/1205.2671 (2012).

4. Battaglieri, M. et al. US cosmic visions: new ideas in dark matter 2017: community report. Preprint at https://arxiv.org/abs/1707.04591 (2017).

5. Lee, B. W., Quigg, C. \& Thacker, H. B. The strength of weak interactions at very-high energies and the Higgs boson mass. Phys. Rev. Lett. 38, 883-885 (1977)

6. Beacham, J. et al. Physics beyond colliders at CERN: beyond the standard model working group report. J. Phys. G 47, 010501 (2019).

7. Batell, B., Pospelov, M. \& Ritz, A. Probing a secluded U(1) at B-factories. Phys. Rev. D 79, 115008 (2009).

8. Meyer, H. B. \& Wittig, H. Lattice QCD and the anomalous magnetic moment of the muon. Prog. Part. Nucl. Phys. 104, 46-96 (2019).

9. Barschel, C. et al. Report from the LHC Fixed Target Working Group of the CERN Physics Beyond Colliders Forum (CERN, 2019).

10. Bursche, A. et al. Physics Opportunities with the Fixed-Target Program of the LHCb Experiment Using an Unpolarized Gas Target (CERN, 2018).

11. Aidala, C. A. et al. The LHCSpin project. Preprint at https://arxiv.org/ abs/1901.08002 (2019)

12. Bagli, E. et al. Electromagnetic dipole moments of charged baryons with bent crystals at the LHC. Eur. Phys. J. C 77, 828 (2017).

13. Krasny, M. W. et al. The gamma factory proposal for CERN. Preprint at https://arxiv.org/abs/1511.07794 (2015) 
14. Krasny, M. W. et al. Gamma factory for CERN, Input \#6 to the European Strategy Update (CERN, 2018)

15. Bartosik, H. et al. SPS Operation and Future Proton Sharing Scenarios for the SHiP Experiment at the BDF Facility (CERN, 2018).

16. Ahdida, C. et al. SPS beam dump facility comprehensive design study. Preprint at https://arxiv.org/abs/1912.06356 (2019).

17. Åkesson et al., T. A primary electron beam facility at CERN. Preprint at https://arxiv.org/abs/1805.12379 (2018).

18. Adli, E. et al. Acceleration of electrons in the plasma wakefield of a proton bunch. Nature 561, 363-367 (2018).

19. Wing, M. et al. Particle physics applications of the AWAKE acceleration scheme. Preprint at https://arxiv.org/abs/1812.11164 (2018).

20. Adey, D. et al. Overview of the neutrinos from stored muons facility - nuSTORM. J. Inst. 12, P07020 (2017).

21. CAST Collaboration. New CAST limit on the axion-photon interaction. Nat. Phys. 13, 584-590 (2017).

22. Siemko, A. et al. PBC Technology Subgroup Report (CERN, 2018).

23. Gazdzicki, A. et al. Study of Hadron-Nucleus and Nucleus-Nucleus Collisions at the CERN SPS: Early Post-LS2 Measurements and Future Plans (CERN, 2018).

24. Agnello, M. et al. Study of hard and electromagnetic processes at CERN-SPS energies: an investigation of the high- $\mu_{\mathrm{B}}$ region of the QCD phase diagram. Preprint at https://arxiv.org/abs/1812.07948 (2018).

25. COMPASS++/AMBER Collaboration. Letter of intent: a new QCD facility at the M2 beam line of the CERN SPS. Preprint at https://arxiv.org/ abs/1808.00848 (2019).

26. Abbiendi, G. et al. Measuring the leading hadronic contribution to the muon g-2 via $\mu$ e scattering. Eur. Phys. J. C 77, 39 (2017)

27. Hadjidakis, C. et al. A fixed-target programme at the LHC: physics case and projected performances for heavy-ion, hadron, spin and astroparticle studies. Preprint at https://arxiv.org/abs/1807.00603 (2018).

28. Cortina Gil, E. et al. First search for $K^{+} \rightarrow \pi^{+} \nu \bar{\nu}$ using the decay-in-flight technique. Phys. Lett. B 791, 156-166 (2019).

29. Ambrosino, F. et al. KLEVER: an experiment to measure $\operatorname{BR}\left(K_{L} \rightarrow \pi^{0} \nu \bar{\nu}\right)$ at the CERN SPS. Preprint at https://arxiv.org/abs/1901.03099 (2019).

30. Ahn, J. K. et al. Search for the $K_{L} \rightarrow \pi^{0} \nu \bar{\nu}$ and $K_{L} \rightarrow \pi^{0} X^{0}$ decays at the J-PARC KOTO experiment. Phys. Rev. Lett. 122, 021802 (2019).

31. Kou, E. et al. The Belle II physics book. Preprint at https://arxiv.org/ abs/1808.10567 (2018).

32. Abusaif, F. et al. Storage ring to search for an electric dipole moment of charged particles - feasibility study. Preprint at https://arxiv.org/ abs/1912.07881 (2019).

33. Chang, S. P. et al. Axion dark matter search using the storage ring EDM method. Phys. Rev. D 99, 083002 (2019).

34. Abel, C. et al. Search for axion-like dark matter through nuclear spin precession in electric and magnetic fields. Phys. Rev. X 7, 041034 (2017)

35. Gninenko, S. et al. Addendum to the NA64 Proposal: Search for the A' $\rightarrow$ invisible and $X \rightarrow e^{+} e^{-}$Decays in 2021 (CERN, 2018).

36. Ahdida, C. et al. SHiP Experiment - Comprehensive Design Study Report CERN, 2019)

37. FASER Collaboration. Technical proposal for FASER: forward search experiment at the LHC. Preprint at https://arxiv.org/abs/1812.09139 (2018).

38. Gligorov, V. V., Knapen, S., Papucci, M. \& Robinson, D. J. Searching for long-lived particles: a compact detector for exotics at LHCb. Phys. Rev. D 97, 015023 (2018).

39. Ball, A. et al. A letter of intent to install a milli-charged particle detector at LHC P5. Preprint at https://arxiv.org/abs/1607.04669 (2016).

40. Alpigiani, C. et al. A Letter of intent for MATHUSLA: a dedicated displaced vertex detector above ATLAS or CMS. Preprint at https://arxiv.org/ abs/1811.00927 (2018).

41. Harland-Lang, L. et al. A fresh look at ALP searches in fixed target experiments. Phys. Lett. B 793, 281-289 (2019).
42. European Strategy for Particle Physics Preparatory Group. Physics briefing book. Preprint at https://arxiv.org/abs/1910.11775 (2019)

43. OSQAR Collaboration. New exclusion limits on scalar and pseudoscalar axionlike particles from light shining through a wall. Phys. Rev. D 92, 092002 (2015).

44. Ruz, J. et al. Next generation search for axion and ALP dark matter with the International Axion Observatory. In: 2018 IEEE Nuclear Science Symposium and Medical Imaging Conference Proc. 10-17 (IEEE, 2018).

45. Bahre, R. et al. Any light particle search II - technical design report. J. Inst. 8 T09001 (2013).

46. Ballou, R. et al. Letter of Intent to Measure Vacuum Magnetic Birefringence: the VMB@CERN Experiment (CERN, 2018).

47. Dainese, A. et al. Physics beyond colliders: QCD working group report. Preprint at https://arxiv.org/abs/1901.04482 (2019).

48. Krasznahorkay, A. J. et al. Observation of anomalous internal pair creation in ${ }^{8}$ Be: a possible indication of a light, neutral boson. Phys. Rev. Lett. 116, 042501 (2016).

49. Krasznahorkay, A. J. et al. New evidence supporting the existence of the hypothetic X17 particle. Preprint at https://arxiv.org/abs/1910.10459 (2019).

50. Feng, J. L. et al. Particle physics models for the $17 \mathrm{MeV}$ anomaly in beryllium nuclear decays. Phys. Rev. D 95, 035017 (2017)

51. Graverini, E., for the ATLAS, CMS and LHCb Collaborations. Flavour anomalies: a review. J. Phys. Conf. Ser. 1137, 012025 (2019).

52. Buttazzo, D., Greljo, A., Isidori, G. \& Marzocca, D. B-physics anomalies: a guide to combined explanations. J. High Energy Phys. 1711, 44 (2017).

53. Bennett, G. W. et al. Final report of the muon E821 anomalous magnetic moment measurement at BNL. Phys. Rev. D 73, 072003 (2006).

54. Pospelov, M. Secluded U(1) below the weak scale. Phys. Rev. D 80, 095002 (2009)

55. Marciano, W. J. et al. Contribution of axion-like particles to lepton dipole moments. Phys. Rev. D 94, 115033 (2016).

56. Bauer, M. et al. Axion-like particles, lepton-flavor violation and a new explanation of $a_{\mu}$ and $a_{e}$. Preprint at https://arxiv.org/abs/1908.00008 (2019).

57. Grange, J. et al. Muon (g-2) technical design report. Preprint at https://arxiv. org/abs/1501.06858v2 (2018)

58. Irastorza, I. G. \& Redondo, J. New experimental approaches in the search for axion-like particles. Prog. Part. Nucl. Phys. 102, 89-159 (2018).

\section{Competing interests}

The authors declare no competing interests.

\section{Additional information}

Correspondence should be addressed to C.V.

Peer review information Nature Physics thanks Maurik Holtrop and the other, anonymous, reviewer(s) for their contribution to the peer review of this work.

Reprints and permissions information is available at www.nature.com/reprints.

Publisher's note Springer Nature remains neutral with regard to jurisdictional claims in published maps and institutional affiliations.

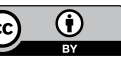

Open Access This article is licensed under a Creative Commons

Attribution 4.0 International License, which permits use, sharing, adaptation, distribution and reproduction in any medium or format, as long as you give appropriate credit to the original author(s) and the source, provide a link to the Creative Commons license, and indicate if changes were made. The images or other third party material in this article are included in the article's Creative Commons license, unless indicated otherwise in a credit line to the material. If material is not included in the article's Creative Commons license and your intended use is not permitted by statutory regulation or exceeds the permitted use, you will need to obtain permission directly from the copyright holder. To view a copy of this license, visit http://creativecommons. org/licenses/by/4.0/

(c) CERN 2020 